\title{
Health Status and Health Care Utilization of Multiple Sclerosis in Canada
}

\author{
Sheri L. Pohar, C. Allyson Jones, Sharon Warren, Karen V.L. Turpin, \\ Kenneth Warren
}

\begin{abstract}
Background: Persons with multiple sclerosis (MS) represent a small segment of the population, but given the progression of the disease, they experience substantial physical, psychosocial and economic burdens. Objective: The primary aim was to compare demographic characteristics, health status, health behaviours, health care resource utilization and access to health care of the community dwelling populations with and without MS. Methods: Cross-sectional survey using data from the Canadian Community Health Survey (CCHS 1.1). Adjusted analyses were performed to assess differences between persons with MS and the general population, after controlling for age and sex. Normalized sampling weights and bootstrap variance estimates were used. Results: Respondents with MS were 7.6 times $(95 \% \mathrm{CI}: 5.4,10.7)$ more likely to have health-related quality of life scores that reflected severe impairment than respondents without MS. Respondents with MS were 12.2 times $(95 \%$ CI: 8.6, 17.2) to rate their health as 'poor' or 'fair' than the general population. Urinary incontinence and chronic fatigue syndrome were 18.7 times (95\% CI: 12.5, 28.2) and 21.9 times (95\% CI: $11.9,40.3)$, more likely to be reported by respondents with MS than those without. Differences between the two populations also existed in terms of health care resource utilization and access and health behaviours. Conclusion: Large discrepancies in health status and health care utilization existed between persons with MS who reside in the community and the general population according to all indicators used. Health care needs of persons with MS were also not met.
\end{abstract}

RÉSUMÉ: Sclérose en plaques, état de santé et utilisation des soins de santé au Canada. Contexte : Les individus atteints de sclérose en plaques (SEP) ne constituent qu'une petite partie de la population, mais ils portent un lourd fardeau physique, psychosocial et économique à cause de la progression de la maladie. Objectif : Le but premier de cette étude était de comparer les caractéristiques démographiques, l'état de santé, les comportements de santé, l'utilisation des ressources de soins de santé et l'accès aux soins de santé des individus vivant dans la collectivité, atteints ou non de la SEP. Méthodes : Il s'agit d'une étude transversale des données de l'Enquête sur la santé dans les collectivités canadiennes (ESCC 1.1). Nous avons analysé les données pour évaluer les différences entre les individus atteints de SEP et la population en général, après ajustement pour l'âge et le sexe. Un échantillonnage normalisé pondéré et l'estimation de variance bootstrap ont été utilisés. Résultats : Les répondants atteints de SEP étaient 7,6 fois (IC de $95 \%: 5,4$ à 10,7) plus susceptibles d'avoir des scores de qualité de vie reliée à la santé qui témoignaient d'une atteinte sévère que les répondants sans SEP. Les répondants atteints de SEP étaient 12,2 fois (IC de 95\%: 8,6 à 17,2) plus susceptibles de décrire leur santé comme étant « mauvaise » ou « passable » que la population en général. Ils étaient 18,7 fois (IC de 95\%: 12,5 à 28,2) plus susceptibles de rapporter de l'incontinence urinaire et 21,9 fois (IC de 95\% : 11,9 à 40,3) plus susceptibles de rapporter un syndrome de fatigue chronique que ceux qui n'en étaient pas atteints. Il y avait également des différences entre les deux populations en termes d'utilisation et d'accès aux ressources de soins de santé et en termes de comportements de santé. Conclusion : Selon tous les indicateurs utilisés, il existe de grandes discordances entre les individus atteints de SEP qui vivent dans la collectivité et la population en général quant à l'état de santé et à l'utilisation des soins de santé. En outre, les besoins de santé des individus atteints de SEP n'étaient pas satisfaits.

Can. J. Neurol. Sci. 2007; 34: 167-174

Multiple sclerosis (MS) is a complex chronic neurological condition typically seen in young adults. Estimated prevalence rates of MS range from 55 to 240 per 100,000 in Canada, ${ }^{1-3}$ making it one of the five most highly prevalent MS countries in the world (Northern US, Northern Europe, Australia, New Zealand). ${ }^{4}$ The associated disability caused by this inflammatory autoimmune disorder of the central nervous system is substantial. Physical and psychosocial dimensions as well as overall quality of life are adversely affected by MS., ${ }^{5,6}$ The array

\footnotetext{
From the Institute of Health Economics (SLP, CAJ); Faculty of Rehabilitation Medicine (CAJ, SW), Faculty of Medicine and Dentistry (KVLT, KW), Edmonton, Alberta, Canada.

RECEIVED OCTOBER 24, 2006. ACCEPTED IN FINAL FORM JANUARY 21, 2007. Reprint requests to: Allyson Jones, Rm 2-50 Corbett Hall, Department of Physical Therapy, University of Alberta, Edmonton, T6G 2G4, Canada.
} 
of disabilities is a major burden not only for patients and families but also for the healthcare system and society. ${ }^{7-9}$

Because of the unpredictable course of disease and variable symptoms, health status in individuals with MS varies. Health related quality of life (HRQL) in patients with MS has been well documented in clinical populations, although less so within population-based studies. ${ }^{10,11}$ Chronic diseases associated with functional disability do not always generate higher use of healthcare resources. ${ }^{12}$ Although a limited amount of evidence evaluating health status and health resource utilization in MS patient population exists, ${ }^{9,13,14}$ little is known about the health status and health resources used by persons with MS who reside in the community as compared to the general population. The use of healthcare resources has particular importance in young patient populations with chronic conditions because of potentially high demands on the healthcare system.

The primary objective of this analysis was to describe and compare health status, health behaviours, health resource utilization, the use of insured medical treatment, and access to health care by persons with MS and the general population.

\section{METHODS}

\section{Survey design and study population}

In this study, health determinants, health status, health care utilization, and access to health care were described based on data collected between September 2000 and November 2001 from the Canadian population. The Canadian Community Health Survey Cycle 1.1 (CCHS 1.1) is a cross-sectional survey that employed a multistage stratified cluster design combined with random sampling methods to select a representative sample of the Canadian population. ${ }^{15}$ The survey excludes individuals living on crown or reserve land, in institutions, members of the Canadian Armed Forces and some remote areas of the country, but still represents approximately $98 \%$ of the Canadian population over 12 years of age. ${ }^{15}$ Approval to access the survey data was obtained from Statistics Canada and ethical approval was obtained through the University of Alberta Health Research Ethics Board.

A total of 131, 535 respondents were surveyed; the overall response rate was $84.7 \% .{ }^{16}$ Of the total sample, 335 respondents reported having a diagnosis of multiple sclerosis; this represented 56,913 Canadians with MS or $0.22 \%$ of the community dwelling Canadian population over the age 12 . The present analysis was restricted to respondents who were 18 years of age or older: 113,916 respondents who did not report MS and 335 who did report having MS.

The CCHS 1.1 includes questions pertaining to various health-related areas such as physical and mental health status, lifestyle behaviours, health care utilization and socioeconomic characteristics. In particular, respondents are asked to report chronic conditions from a list of 27 conditions, one of them being MS. Identification of all chronic conditions is based upon a self-reported diagnosis, with the exception of depression. Although the validity of self-reported diagnoses varies, the questionnaire stipulates that chronic conditions be diagnosed by a health professional and be present for at least six months or be expected to last for at least six months.

\section{Health determinants}

A broad range of dimensions were included when considering health determinants. ${ }^{17}$ Age, sex, marital status, country of birth (Canada or other), race, socioeconomic status and employment status within the past 12 months were compared between respondents with and without MS. Socioeconomic status was delineated using three variables: highest level of education, receipt of social assistance as income source and food insecurity. Three questions were used to define food insecurity in terms of lacking financial access to a sufficient quantity and quality of food. ${ }^{16}$

\section{Health status}

A number of indicators were used to define health status: HRQL; self-rated health; self-rated health compared to one-year ago; chronic medical conditions; number of disability days (the number of days in the last two weeks when the respondent remained in bed or reduced activities because of illness or injury); impact of health problems; difficulty with activities; and need for assistance with one or more instrumental activity of daily living (IADL) such as preparing meals, shopping for necessities, doing daily housework, performing personal care and indoor mobility. In addition to examining the individual chronic medical conditions, a summative score of the total number of chronic conditions was also generated as a disability indicator.

Health-related quality of life was measured using the Health Utilities Index Mark 3 (HUI3), a generic preference-based measure. ${ }^{18}$ HUI3 health states are defined by eight of attributes (vision, hearing, speech, ambulation, dexterity, emotion, cognition and pain and discomfort), with 5 or 6 levels of functioning for each attribute. A utility function is used to obtain an overall score for health states that range from -0.36 to 1.0 ($0.36=$ worst possible health, $0.0=$ dead and $1.0=$ perfect health). ${ }^{18}$ A difference of 0.03 between overall HUI3 scores is considered to be clinically important. ${ }^{19}$ Overall HUI3 scores can also be grouped into categories reflecting level of impairment: none/mild (0.89 to 1.00$)$, moderate $(0.70$ to 0.88$)$ and severe (less than 0.70). ${ }^{20}$ Self-rated health was assessed on a 5-point Likert scale (poor to excellent health), as was self-rated health compared to one year ago (much worse to much improved).

The Composite International Diagnostic Interview Short Form for Major Depression (CIDI-SFMD) was used to assess the probability of a major depressive disorder. A probability of 0.90 is consistent with a diagnosis of Major Depressive Disorder in accordance with the DSM-IV diagnostic criteria. ${ }^{21}$ This is a brief survey instrument which was derived from the $\mathrm{CIDI}^{22}$ and subsequently has been used by Statistics Canada for the health surveys.

Impact of health problems, that is, the degree to which longterm health problems impacted home, work/ school, and other activities, as well as difficulty with activities of daily living (ADL) and IADL was assessed in the survey with a number of questions, from which three categorical response options (never, sometimes, often) were derived. ${ }^{16}$ 


\section{Health behaviours}

Self-reported smoking status, alcohol consumption, and physical activity level were classified as health behaviours. Respondents who consumed five drinks or more on one occasion more than once a month were considered heavy drinkers. ${ }^{23}$ Categorization of respondents' physical activity level was based upon energy expenditure, using guidelines from previous health surveys. ${ }^{16,24}$

\section{Health care resource utilization}

The Canadian health care system is a single-payer which ensures that Canadian citizens are fully insured and provided with universal access for hospital and medical services. Respondents reported number of physician visits, overnight hospitalizations and consultations with health professionals over the previous 12 months. Access to medical care was determined within the CCHS 1.1 by self-perceived unmet healthcare needs and whether respondents had a regular medical doctor.

\section{Analysis}

Descriptive statistics were used to summarize the characteristics of the two populations, those with MS and the general population. To assess the significance of differences of proportions between groups the chi square test $\left(\chi^{2}\right)$ was used; the t-test was used for continuous data. When less than $10.0 \%$ of respondents with MS reported chronic conditions, coefficients of variation for these estimates exceeded the acceptable limits suggested by Statistics Canada and, as such, are not reported. ${ }^{16}$

Adjusted analyses were performed to further assess the differences between the two groups in terms of health status, health behaviours, health care resource utilization, and access to health care resources, after adjusting for age and sex. Analysis of covariance was used for comparisons made for continuous variables and logistic regression analysis was used for comparisons of dichotomous variables. Categories were collapsed to create dichotomous outcomes for categorical variables with more than two response options. Because of the complex sampling design, sampling weights were applied to all analyses in order to account for the unequal probability of being selected into the survey. ${ }^{16}$ Bootstrap variance estimates derived by Statistics Canada were used to adjust for clustering and stratification ${ }^{16}$ and to estimate 95 percent confidence intervals (95\% CI) and p-values. All analyses were performed using WESTVAR, version 4.2.

\section{RESUlts}

As seen in Table 1, persons with MS tended to be older, female, Caucasian, and Canadian born than the general population without MS. Although the level of education was

Table 1: Demographic characteristics of respondents with and without MS

Population with MS

General Population

(without MS)

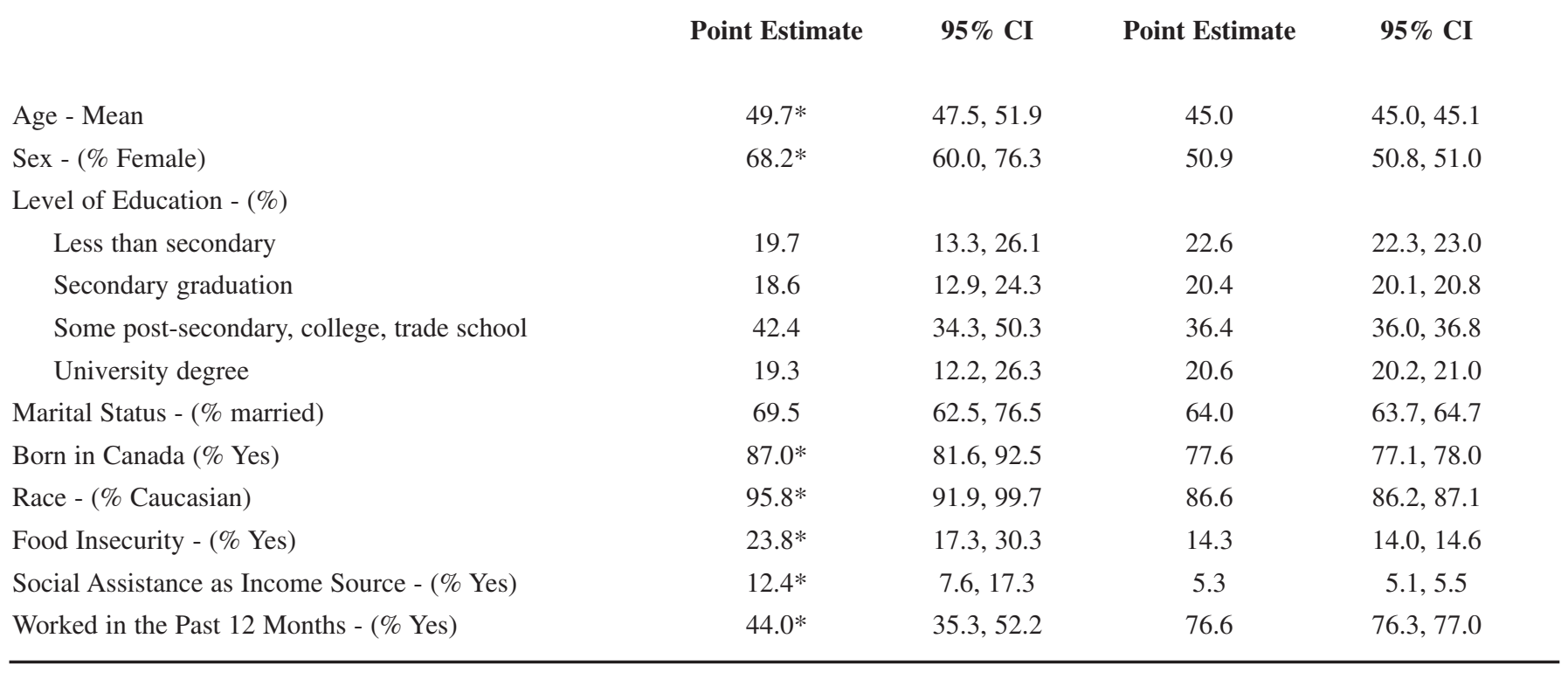

$* \mathrm{p}<0.05$ compared to general population 
Table 2: Health status, healthcare behaviours, health care resource utilization and access to health care of respondents with MS and the general population without MS

Population with MS

\section{Health Status}

Overall HUI3 Score (Mean)

HUI3 Disability Categories (\%)

No or mild impairment

Moderate impairment

Severe Impairment

Self-Rated Health (\%)

Excellent

Very good

Good

Fair

Poor

Health compared to one year ago (\%)

Much better

Somewhat better

About the same

Somewhat worse

Much worse

Impact of health problems (\%)

Often

Sometimes

Never

Has difficulty with activities (\%)

Often

Sometimes

Never

Needs assistance with one or more task (\%)

Number of disability days - Mean

Urinary Incontinence (\%)

Depression (\%)

Hypertension (\%)

Arthritis (\%)

Allergies other than food (\%)

Back Problems (\%)

Migraine (\%)

Chronic Fatigue Syndrome (\%)

Health Behaviours

Physical activity (\%)

Active

Moderately active

Inactive

Type of Smoker (\%)

Daily

Occasionally

Not at all

Heavy Drinker - (\%)

Health Care Resource Utilization

Overnight patient - (\% Yes $)$

Consulted a mental health professional (\% Yes)

Number of consultations with medical doctors

(Mean)

Access to Health Care

Has a regular medical doctor - (\% Yes)

Self-perceived unmet healthcare needs - $(\%$ Yes $)$
Point Estimate

$0.55^{*}$

$30.5^{*}$

14.4

$55.0^{*}$

$2.2^{*}$

$6.5^{*}$

28.5

$36.3 *$

$26.5^{*}$

2.7 *

13.9

$48.6^{*}$

$26.3^{*}$

$8.6^{*}$

$52.0^{*}$

$35.5^{*}$

$12.5 *$

$50.8^{*}$

$26.2 *$

$22.9 *$

$72.3^{*}$

$3.6^{*}$

$28.7 *$

$15.7 *$

17.1

26.4*

29.2

$34.5 *$

14.2

17.1 *

$7.6^{*}$

18.9

$73.5^{*}$

$31.9 *$

4.7

$63.4 *$

$3.5^{*}$

$20.8^{*}$

$20.0^{*}$

$8.4^{*}$

96.4*

$26.0 *$
$95 \% \mathrm{CI}$

$0.49,0.61$

$22.8,38.3$

8.1, 20.7

47.0, 63.1

$0.8,3.7$

3.6, 9.5

$21.2,35.8$

$28.4,44.3$

$19.5,33.4$

0.6, 4.7

$7.8,19.9$

$40.6,56.5$

$20.2,32.4$

$3.8,13.5$

44.1, 59.8

27.4, 43.6

7.4, 17.7

$43.4,58.2$

$19.9,32.6$

$15.5,30.3$

66.1, 78.6

2.7, 4.5

$21.1,36.2$

$10.8,20.6$

$10.6,23.6$

19.2, 33.6

$22.1,36.3$

26.6, 42.5

$8.3,20.2$

9.7, 24.5

$4.3,10.9$

$11.2,26.6$

61.6, 81.4

$24.6,39.3$

$1.9,7.5$

$55.8,70.9$

1.1, 5.8

$14.3,27.1$

$13.4,26.5$

7.1 to 9.6

$94.1,98.7$

$18.9,33.1$
General Population

(without MS)

Point Estimate

$95 \% \mathrm{CI}$

0.87

$0.87,0.88$

72.2

14.6

$71.8,72.5$

$14.3,14.9$

$12.9,13.5$

25.2

$24.7,25.5$

$34.9,35.7$

$26.5,27.2$

$9.2,9.7$

3.1, 3.4

$6.2,6.6$

$10.5,10.9$

$70.8,71.5$

$9.7,10.2$

1.7, 1.9

$10.3,10.8$

$12.9,13.5$

$75.8,76.6$

$10.8,11.3$

$14.0,14.6$

$74.2,75.0$

$14.0,14.6$

$0.95,1.00$

$2.2,2.4$

7.2, 7.6

13.6, 14.2

$16.5,17.1$

24.7, 25.4

$18.5,19.1$

$9.2,9.7$

$0.8,0.9$

0.8

20.7

23.5

55.9

$20.3,21.0$

$23.0,23.8$

$55.4,56.4$

$22.4,23.1$

$4.2,4.6$

$72.4,73.2$

$10.5,11.0$

72.8

10.8

$(8.4,8.8)$

8.6

$(8.3,8.8)$

4.5

(4.4 to 4.5$)$

83.8

$(83.5,84.2)$

$(12.7,13.3)$

$* \mathrm{p}<0.05$ compared to general population; $\dagger$ Unadjusted 
similar between the two groups, individuals with MS were more likely to receive social assistance $(12.4 \%$, 95\% CI 7.6, 17.3 versus $5.3 \%, 95 \%$ CI 5.1, 5.5) and experience food insecurity in the previous 12 months $(23.8 \%, 95 \%$ CI 17.3, 30.0 versus $14.3 \%, 95 \%$ CI 14.0, 14.6). A substantially lower proportion of the MS population had worked in the previous 12 months compared to the population without MS $(44.0 \%, 95 \%$ CI 35.3, 52.2 versus $76.6 \%, 95 \%$ CI $76.3,77.0$ ).

\section{Health status}

Without adjustment for age and sex, the health status of respondents with MS was significantly impaired compared to respondents without MS (Table 2). Fifty-five percent (95\% CI $47.0,63.1)$ of the MS population experienced severe impairment as defined by the HUI3 disability classification system; $13 \%$ (95\% CI 12.9, 13.5) of the population without MS reported severe impairment. Additionally, 'poor' self-rated health was reported by $26.5 \%(95 \%$ CI $19.5,33.4)$ of the MS population compared to $3.2 \%(95 \%$ CI $3.1,3.4)$ of the population without MS. Health problems more often impacted the lives of persons with MS; difficulties with activities and requiring assistance with tasks were also more frequently reported. As a further indicator of health status, a number of chronic conditions were more frequently reported by respondents with MS (Table 2).

Results of the adjusted analysis were consistent with the bivariate analyses. After adjustment for age and sex, respondents with MS had a lower average overall HUI3 score than the general population. The mean difference $(0.31$ [95\% CI: 0.25 , 0.37]) was more than ten times the clinically important difference. Compared to the population without MS, respondents with MS were $7.6(95 \%$ CI: $5.4,10.7)$ times more likely to have overall HUI3 scores in the severe impairment range (Table 3). Similarly, respondents with MS were 12.2 (95\% CI: 8.6, 17.2) times more likely to rate their health as 'poor' or 'fair' than

Table 3: Age and sex adjusted comparison of health status, health behaviours, health care resource utilization and access to health care of respondents with and without MS

\section{Health Status \\ HUI3 Disability Categories Severe impairment \\ Self-Rated Health Poor to fair \\ Health compared to one year ago Somewhat worse to much worse \\ Impact of health problems Often \\ Has difficulty with activities Often}

Needs Assistance with at least one task

Urinary Incontinence

Depression

Hypertension

Arthritis

Allergies other than food

Back Problems

Migraine

Chronic Fatigue Syndrome

\section{Health Behaviors}

Physical activity Inactive

Type of Smoker Daily

Heavy Drinker

Health Care Resource Utilization

Overnight patient

Consulted a mental health professional

\section{Access to Health Care}

Has a regular medical doctor

Self-perceived unmet healthcare

\section{Reference Group}

No to moderate impairment

Good to excellent

About the same to much better

Sometimes to never

Sometimes to never

$$
\text { No }
$$

No

No

No

No

No

No

No

No

Moderately active to
active

Occasionally to not at all No

No

No

No

No

Adjusted $^{\mathrm{A}}$ Odds Ratio

95\% CI

7.6

12.2

3.7

8.9

8.5

17.9

18.7

2.3

1.1

1.5

1.2

2.2

1.5

21.9

2.0

1.9

0.5

2.5

2.6

3.8

2.4
$5.4,10.7$

$8.6,17.2$

2.7, 5.0

$6.3,12.6$

6.2, 11.6

$12.3,26.1$

$12.5,28.2$

$1.6,3.4$

$0.6,1.9$

$1.0,2.2$

$0.8,1.7$

$1.5,3.1$

$0.9,2.5$

$11.9,40.3$

$1.4,3.0$

$1.3,2.6$

$0.2,1.1$

$1.7,3.7$

1.7, 3.9

$1.8,7.9$

$1.7,3.5$ 
respondents without MS. They were also more likely to report that health problems often impacted their lives (odds ratio $(\mathrm{OR})=8.9 ; 95 \% \mathrm{CI}: 6.3,12.6)$ and specify a deterioration of health $(\mathrm{OR})=3.7 ; 95 \% \mathrm{CI}: 2.7,5.0)$ than the general population (Table 3).

Respondents with MS were more likely to have a number of chronic conditions. After adjustment, those with MS appeared to be at particular risk for urinary incontinence and chronic fatigue syndrome, being 18.7 times (95\% CI: 12.5, 28.2) and 21.9 times (95\% CI: 11.9, 40.3) more likely to report these conditions, respectively, than respondents without MS (Table 3).

\section{Health behaviours}

After adjusting for age and sex, respondents with MS were 2.0 times (95\% CI: $1.4,3.0)$ more likely to be inactive and 1.9 times $(95 \%$ CI: $1.3,2.6)$ more likely to smoke than respondents without MS (Table 3). Consistent with their relatively poor health status, respondents with MS had an adjusted average of 2.5 (95\% CI: 1.6, 3.4) more disability days than respondents without MS in the previous 12 months.

\section{Health care resource utilization}

Use of health care resources within the MS population clearly exceeded the resources of the population without MS (Tables 2 and 3). After adjustment, respondents with MS were more than twice as likely to be an overnight patient or consult a mental health professional as compared to respondents without MS. More consultations with medical doctors in the previous 12 months were reported by respondents with MS than respondents without MS (adjusted mean difference $=3.4 ; 95 \%$ CI: 2.2, 4.7). The probability of respondents with MS having unmet health care needs was 2.4 times $(95 \%$ CI: $1.7,3.5)$ that of respondents without MS, despite being more likely to have a regular medical doctor (Table 3).

\section{DISCUSSION}

Using data from a nationally representative community population-based sample, large disparities in health status, health behaviours, health care resource utilization and access to health care were observed between respondents with and without MS. According to all indicators used, the health status of individuals with MS was significantly impaired relative to those without. Although the level of impairment observed in the MS population is consistent with small clinic samples; the findings from this study compared this level of impairment to the general population.

The observed impairment of the HUI3 and self-rated health are particularly noteworthy. Individuals with MS were almost eight times more likely to have scores that reflected severe impairment on the HUI3 than individuals without MS. In a Canadian population-based study, the average overall HUI3 score individuals with diabetes, heart disease, arthritis or stroke ranged from 0.74 to $0.89 .{ }^{25}$ Overall HUI3 scores of individuals with three of these conditions in any combination ranged from 0.62 to $0.66^{25}$ which is still higher than the overall HUI3 score (0.55) for our MS sample. Thus, the burden associated with MS was substantially higher than with diabetes, heart disease, arthritis or stroke alone or in combination. This highlights the extreme impairment that can be associated with MS, compared to community dwelling individuals with multiple chronic conditions.

Consistent with results observed for the HUI3, almost twothirds of respondents with MS rated their health as 'fair' or 'poor' and were 12 times more likely to report 'fair' or 'poor' health than the general population without MS. A previous study based on CCHS 1.1 data found that approximately $42 \%$ of respondents with type 2 diabetes rated their health as 'fair' or 'poor', ${ }^{26}$ a much lower proportion than respondents with MS reported in the present study. This is somewhat surprising given that the Canadian population with type 2 diabetes is substantially older than the MS population and is affected by a number of age and diabetes-related comorbidities. ${ }^{26}$ Despite this, the self-rated health of individuals from the younger MS population was worse, again emphasizing the burden of MS compared to other chronic conditions.

Chronic conditions were associated with significantly impaired health status and also appear to be associated with increased utilization of health care resources. ${ }^{26-29}$ We found that individuals with MS were more likely to have back problems, depression, arthritis and urinary incontinence than the population without MS. Although depression, ${ }^{30}$ urinary incontinence, ${ }^{31}$ and back problems ${ }^{32-35}$ have been reported in MS, few have compared these conditions in a community-based population. Comorbid depression and back pain, specifically, have been shown to increase health care resource utilization. ${ }^{26,29,36,37}$ Depression and fatigue have been reported by others to negatively impact HRQL in patients with MS. ${ }^{5,38}$

The frequency of chronic fatigue syndrome warrants discussion given the clinical context of MS. Fatigue has been reported as one of the most prevalent and distressing symptoms of MS. ${ }^{39,40}$ Although validation of each condition within this survey was based on the diagnosis of a health professional and the chronicity of a minimum of six months, it is most likely that respondents acknowledged fatigue associated with MS and did not differentiate it from the clinical definition of Chronic Fatigue Syndrome.

When the impact of 21 conditions was assessed, urinary incontinence had the largest impact on HRQL in younger respondents, that is, respondents who were under the age of 44 years. In relative terms, urinary incontinence was reported to have the third most negative impact on overall impairment across the Canadian general population, behind only Alzheimer's disease and stroke. ${ }^{41}$

The burden of the MS population was also reflected in measures of disability. Over one-half reported often having difficulties with activities and almost three-quarters required assistance with one or more tasks. Our findings of inactivity reported within this population concur with Motl and colleagues who completed a meta-analysis of physical activity in persons with MS. ${ }^{42}$ They reported persons with MS were significantly less active than either healthy or disease comparison groups, irrespective of objective or self-report measures of physical activity. ${ }^{42}$ In our study, inactivity was further captured by the average number of disability days (i.e., the number of days in the last two weeks when the respondent stayed in bed or reduced the 
number of activities because of illness or injury). The number of disability days was considerably higher in the MS population than the general population.

Given the association between HRQL and health care resource utilization, ${ }^{43-47}$ it is not surprising that individuals with MS used more health care resources. Our findings indicated that the health care needs of persons with MS were not being met. Although the vast majority of respondents with MS (96.4\%) had a regular medical doctor, this did not guarantee that healthcare needs were met. As unmet health care needs increased in the Canadian population between the 1995 and 2001, features of the healthcare system are cited as the reason for these unmet needs. ${ }^{48}$ This finding is important for health policy makers to consider, given that reasons often cite that unmet healthcare needs are beyond the control of the patient and clinician such as excessive wait times and unavailability of services within an area. ${ }^{48}$ At the same time, it is important to note that unmet health care needs have been associated with poor health status and even death. ${ }^{49,50}$ Thus, it is essential that health care needs of both the population with and without MS are being met in order to improve or maintain the population's health status.

The strengths of this study are noteworthy. In particular, findings were based on a national population-based survey with a high response rate $(84 \%)$. The breadth of content covered in the CCHS 1.1 allowed for comparison of the MS and general population on multiple measures of health determinants, health status, health behaviours, health care utilization and access to health care in a single study. Limitations should be recognized within the context of these findings. Ascertainment of MS and other chronic conditions was via self-report. Although questions regarding the presence of medical conditions specified that the condition be diagnosed by a health professional, there remained potential for individuals to over- or under-report any medical condition, including MS. Healthcare resource utilization was also self-reported and relied on recall over the previous year.

Health behaviours (e.g. alcohol use, level of physical activity and smoking status) were self-reported and questions may have been answered in a socially desirable manner. The validity of self-reported level of physical activity may be questioned by some, but it should be emphasized that the physical activity index was based on detailed responses to 47 questions on participation in specific activities and participation in other activities. This reduced the need to recall the activities in which the respondents participated; however, duration of time spent in each activity may have been subject to inaccurate recall or social desirability.

Another limitation is the cross sectional survey design which prevents the examination of causal effects. Regardless of this limitation, the cross-sectional relationships among health states, health care resource utilization and access to health services provided insight as to the HRQL and services available to persons with MS residing in the community.

Despite over $98 \%$ of the Canadian community dwelling population being represented in the survey, the generalizability of the results to the entire Canadian population with MS is limited by the fact that those individuals who reside in institutions were not captured by the sampling frame. Thus, the results are only generalizable to the community dwelling population with MS. That being said, for some comparisons, such as HUI3 scores, self-rated health, difficulties with activities, and need for assistance with tasks, it is likely that those individuals with MS who reside in institutions would have reported poorer health status and greater impairment. Thus, the true burden of entire Canadian population with MS residing within both community and institutional facilities would be under-estimated by these results.

In conclusion, our findings quantify the poor health status, excess health care utilization and unmet healthcare needs of the community dwelling population with MS in comparison to the general population. Individuals with MS represent a small segment of the community, but are particularly burdened. The magnitude of the differences observed between MS and the general population draws attention and further research to the disparities in healthcare for the MS population.

\section{ACKNOWLEDGEMENTS}

The research and analysis are based on data from Statistics Canada. The opinions expressed do not represent the views of Statistics Canada.

\section{REFERENCES}

1. Pryse-Phillips WE. The incidence and prevalence of multiple sclerosis in Newfoundland and Labrador, 1960-1984. Ann Neurol. 1986; 20(3):323-8.

2. Beck CA, Metz LM, Svenson LW, Patten SB. Regional variation of multiple sclerosis prevalence in Canada. Mult Scler. 2005; 11(5):516-19.

3. Rosati G. The prevalence of multiple sclerosis in the world: an update. Neurol Sci. 2001; 22(2):117-39.

4. Warren S, Warren KG. Multiple Sclerosis. Geneva: World Health Organization; 2001.

5. Lobentanz IS, Asenbaum S, Vass K, Sauter C, Klosch G, Kollegger $\mathrm{H}$, et al. Factors influencing quality of life in multiple sclerosis patients: disability, depressive mood, fatigue and sleep quality. Acta Neurol Scand. 2004; 110(1):6-13.

6. Lublin FD, Reingold SC. Defining the clinical course of multiple sclerosis: results of an international survey. National Multiple Sclerosis Society (USA) Advisory Committee on Clinical Trials of New Agents in Multiple Sclerosis. Neurology. 1996; 46(4):907-11.

7. Pompeii LA, Moon SD, McCrory DC. Measures of physical and cognitive function and work status among individuals with multiple sclerosis: a review of the literature. J Occup Rehabil. $2005 ; 15(1): 69-84$

8. Patwardhan MB, Matchar DB, Samsa GP, McCrory DC, Williams RG, Li TT. Cost of multiple sclerosis by level of disability: a review of literature. Mult Scler. 2005; 11(2):232-9.

9. Burden of illness of multiple sclerosis: Part I: Cost of illness. The Canadian Burden of Illness Study Group. Can J Neurol Sci. 1998; 25(1):23-30.

10. Pittock SJ, Mayr WT, McClelland RL, Jorgensen NW, Weigand SD, Noseworthy JH, et al. Quality of life is favorable for most patients with multiple sclerosis: a population-based cohort study. Arch Neurol. 2004; 61(5):679-86.

11. Nortvedt MW, Riise T, Myhr KM, Nyland HI. Quality of life in multiple sclerosis: measuring the disease effects more broadly. Neurology. 1999; 53(5):1098-103.

12. Verbrugge LM, Patrick DL. Seven chronic conditions: their impact on US adults' activity levels and use of medical services. Am J Public Health. 1995; 85(2):173-82.

13. Hardiman O, Corr B, Frost E, Gibbons P, Mahon L, Traynor BJ. Access to health services in Ireland for people with Multiple Sclerosis and Motor Neurone Disease. Ir Med J. 2003; 96(7): 200-3. 
14. Stolp-Smith KA, Atkinson EJ, Campion ME, O'Brien PC, Rodriguez M. Health care utilization in multiple sclerosis: a population-based study in Olmsted County, MN. Neurology. 1998; 50(6):1594-600.

15. Beland Y. Canadian community health survey--methodological overview. Health Rep. 2002; 13(3):9-14.

16. Statistics Canada. CCHS Cycle 1.1, Public Use Microdata File Documentation; 2004.

17. Evans RG, Stoddart GL. Producing health, consuming health care. Soc Sci Med. 1990; 31(12):1347-63.

18. Feeny D, Furlong W, Torrance GW, Goldsmith CH, Zhu Z, Depauw $\mathrm{S}$, et al. Multiattribute and single-attribute utility functions for the health utilities index mark 3 system. Med Care. 2002; 40(2): $113-28$

19. Horsman J, Furlong W, Feeny D, Torrance G. The Health Utilities Index (HUI(R)): concepts, measurement properties and applications. Health Qual Life Outcomes. 2003; 1(1):54.

20. Health Utilities Inc. [Cited 2004 Aug 20]. Available from http://www.healthutilities.com.

21. Patten SB, Brandon-Christie J, Devji J, Sedmak B. Performance of the composite international diagnostic interview short form for major depression in a community sample. Chronic Dis Can. 2000; 21(2):68-72.

22. Wittchen HU. Reliability and validity studies of the WHO-Composite International Diagnostic Interview (CIDI): a critical review. J Psychiatr Res. 1994; 28(1):57-84.

23. Shields M, Tremblay S. The health of Canada's communities. Health Rep. 2002; 13 Suppl:1-23.

24. Campbell Survey on Well-Being in Canada. [Cited 2005 Aug 10]. Available from: http://cflri.ca/cflri/pa/surveys/88survey.html.

25. Maddigan SL, Feeny DH, Johnson JA. Health-related quality of life deficits associated with diabetes and comorbidities in a Canadian National Population Health Survey. Qual Life Res. 2005; 14(5):1311-20.

26. Maddigan S, Feeny D, Majumdar S, Farris K, Johnson J. Construct validity of the Health Utilities Index Mark 3 in type 2 diabetes: evidence from a nationally representative sample of Canadians. Institute of Health Economics Working Paper. WP 05-02; 2005.

27. Simpson SH, Corabian P, Jacobs P, Johnson JA. The cost of major comorbidity in people with diabetes mellitus. CMAJ. 2003; 168(13):1661-7.

28. Sprenkle MD, Niewoehner DE, Nelson DB, Nichol KL. The Veterans Short Form 36 questionnaire is predictive of mortality and health-care utilization in a population of veterans with a selfreported diagnosis of asthma or COPD. Chest. 2004; 126(1): 81-9.

29. Cramer JA, Blum D, Fanning K, Reed M. The impact of comorbid depression on health resource utilization in a community sample of people with epilepsy. Epilepsy Behav. 2004; 5(3):337-42.

30. Dalton EJ, Heinrichs RW. Depression in multiple sclerosis: a quantitative review of the evidence. Neuropsychology. 2005; 19(2):152-8.

31. Wollin J, Bennie M, Leech C, Windsor C, Spencer N. Multiple sclerosis and continence issues: an exploratory study. Br J Nurs. 2005; 14(8):439-6.

32. Svendsen KB, Jensen TS, Overvad K, Hansen HJ, Koch-Henriksen N, Bach FW. Pain in patients with multiple sclerosis: a population-based study. Arch Neurol. 2003; 60(8):1089-94.
33. Kalia LV, O'Connor PW. Severity of chronic pain and its relationship to quality of life in multiple sclerosis. Mult Scler. 2005; 11(3):322-7.

34. Solaro C, Brichetto G, Amato MP, Cocco E, Colombo B, D'Aleo G, et al. The prevalence of pain in multiple sclerosis: a multicenter cross-sectional study. Neurology. 2004; 63(5):919-21.

35. Rae-Grant AD, Eckert NJ, Bartz S, Reed JF. Sensory symptoms of multiple sclerosis: a hidden reservoir of morbidity. Mult Scler. 1999; 5(3):179-83.

36. Pearson SD, Katzelnick DJ, Simon GE, Manning WG, Helstad CP, Henk HJ. Depression among high utilizers of medical care. J Gen Intern Med. 1999; 14(8):461-8.

37. Luo X, Pietrobon R, Sun SX, Liu GG, Hey L. Estimates and patterns of direct health care expenditures among individuals with back pain in the United States. Spine. 2004; 29(1):79-86.

38. Amato MP, Ponziani G, Rossi F, Liedl CL, Stefanile C, Rossi L. Quality of life in multiple sclerosis: the impact of depression, fatigue and disability. Mult Scler. 2001; 7(5):340-4.

39. Koch LC, Rumrill PD, Jr., Roessler RT, Fitzgerald S. Illness and demographic correlates of quality of life among people with multiple sclerosis. Rehabil Psychol. 2001; 46(2):154-64.

40. Aronson KJ. Quality of life among persons with multiple sclerosis and their caregivers. Neurology. 1997; 48(1):74-80.

41. Schultz SE, Kopec JA. Impact of chronic conditions. Health Rep. 2003; 14(4):41-53.

42. Motl RW, McAuley E, Snook EM. Physical activity and multiple sclerosis: a meta-analysis. Mult Scler. 2005; 11(4):459-63.

43. Spertus JA, Jones P, McDonell M, Fan V, Fihn SD. Health status predicts long-term outcome in outpatients with coronary disease. Circulation. 2002; 106(1):43-9.

44. Walter-Ginzburg A, Chetrit A, Medina C, Blumstein T, Gindin J, Modan B. Physician visits, emergency room utilization, and overnight hospitalization in the old-old in Israel: the crosssectional and longitudinal aging study (CALAS). J Am Geriatr Soc. 2001; 49(5):549-56.

45. Fan VS, Curtis JR, Tu SP, McDonell MB, Fihn SD. Using quality of life to predict hospitalization and mortality in patients with obstructive lung diseases. Chest. 2002; 122(2):429-36.

46. Brown EM, Goel V. Factors related to emergency department use: results from the Ontario Health Survey 1990. Ann Emerg Med. 1994; 24(6):1083-91.

47. Ethgen O, Kahler KH, Kong SX, Reginster JY, Wolfe F. The effect of health related quality of life on reported use of health care resources in patients with osteoarthritis and rheumatoid arthritis: a longitudinal analysis. J Rheumatol. 2002; 29(6):1147-55.

48. Sanmartin C, Houle C, Tremblay S, Berthelot JM. Changes in unmet health care needs. Health Rep. 2002; 13(3):15-21.

49. Maddigan SL, Feeny DH, Majumdar SR, Farris KB, Johnson JA. Understanding the determinants of health for people with type 2 diabetes. Am J Public Health. 2006; 96(9):1649-55.

50. Alonso J, Orfila F, Ruigomez A, Ferrer M, Anto JM. Unmet health care needs and mortality among Spanish elderly. Am J Public Health. 1997; 87(3):365-70. 